\title{
GeV Gamma-Ray Sources
}

\author{
R.C. Lamb ${ }^{1}$ \& D.J. Macomb ${ }^{2,3}$ \\ ${ }^{\prime}$ Space Radiation Laboratory, California Institute of Technology, Pasadena, CA 91125, USA \\ ${ }^{2}$ Astronomy Programs, Universities Space Research Association, USA \\ ${ }^{3}$ Laboratory for High Energy Astrophysics, NASA/GSFC, Greenbelt, MD 2077I, USA
}

\begin{abstract}
We report on the preliminary extension of our work on cataloging the GeV sky to approximately 7 years of CGRO/EGRET observations with special emphasis on a search for transient sources. The search method and significance levels are presented. Our initial results on 13 possible transients indicate that 3 may be new gamma-ray sources. Sixteen new steady GeV sources are also detected, 3 of which have never been reported as gamma-ray sources.
\end{abstract}

\section{INTRODUCTION}

The sources detected by EGRET above $100 \mathrm{MeV}$ are described in a series of papers culminating in the $3^{\text {rd }}$ EGRET catalog (Hartman et al. 1999). There are several TeV sources which are not listed in the EGRET catalogs such as MRK 501 [1], 1ES $2344+514$ [2], and SN 1006 [3]. Both blazars and supernova remnants can therefore have spectral shapes which make them preferentially detected in certain parts of a very broad gamma-ray energy range (the high-energy portion of which extends from 100 $\mathrm{MeV}$ to at least $10 \mathrm{TeV}$ ). This leads us to fully investigate the EGRET database above $1 \mathrm{GeV}$, an order of magnitude above the threshold for the standard EGRET catalogs.

We divide our search into two separate parts; a search for new steady sources and a search for $\mathrm{GeV}$ transients. Note that steady in this context means detectable in the full all-sky data, not showing a lack of variability. Our data consists of 99000 photons with energies above $1 \mathrm{GeV}$ spread over 217 publicly available EGRET data sets taken over $71 / 2$ years. We begin by extending our list of steady $\mathrm{GeV}$ sources beyond the catalog of Lamb and Macomb ([4], hereafter LM97). These sources then form the basis for our search for transients.

\section{NEWLY DISCOVERED STEADY SOURCES}

Finding transient sources requires understanding steady sources. Although this $\mathrm{GeV}$ photon database is only $10 \%$ large than in LM97, there is evidence for new sources. In addition, the $3^{\text {rd }}$ EGRET Catalog [5] provides insight into weak source candidates. We prepared a database of nearly 99000 photons in maps that represent the full EGRET database through the early CGRO cycle 7 (Viewing period 710). The data preparation and treatment is as described in LM97. Briefly, photons above $1 \mathrm{GeV}$

CP515, GeV-TeV Gamma Ray Astrophysics Workshop, edited by B. L. Dingus, et al. (C) 2000 American Institute of Physics $1-56396-938-6 / 00 / \$ 17.00$ 
are binned in $0.5 \times 0.5$ degree all-sky maps then analyzed using maximum likelihood [6] and the diffuse model of galactic emission [7]. Sources detected above a set threshold are added to the background model and residual likelihood maps are prepared which allow a search for successively weaker sources.

Simulations of twelve all-sky maps using actual exposure indicates that a threshold of $4.5 \sigma$ ensures that sources found in the full EGRET database are real. The number of spurious sources at this significance level is expected to be less than one. Ten sources beyond those in LM97 exceed this threshold. In addition to sources detected above $4.5 \sigma$, we include sources with significance above $4 \sigma$ that have a $100 \mathrm{MeV}$ counterpart in the $3^{\text {rd }}$ EGRET catalog. This counterpart is defined by having the $95 \% \mathrm{GeV}$ and $100 \mathrm{MeV}$ error circles overlapping. Four new GeV sources are in this category. A third category of source are those whose significance is above $4 \sigma$, and are detected at a significance of at least $3 \sigma$ in the $0.3-1.0 \mathrm{GeV}$ energy band at exactly the position of the $\mathrm{GeV}$ excess (supported by simulations). Two such cases exist. Following these rules, Table 1 lists the steady GeV gamma-ray sources beyond those of LM97.

Table 1. New sources found in the full EGRET database, ordered by significance. For sources below 4.5 $\sigma$, the qualifying characteristic is noted (3EG counterpart or probable $0.3-1.0 \mathrm{GeV}$ detection).

\begin{tabular}{|c|c|c|c|c|c|c|}
\hline Name & lii & bii & Sigma & Flux $^{A}$ & $\operatorname{Rad}^{\mathbf{B}}$ & Notes \\
\hline GEV J2159-3024 & $\overline{17.45}$ & -52.32 & 6.3 & $1.8 \pm 0.5$ & 33 & $\begin{array}{l}\text { 3EG J2158-3023, PKS 2155- } \\
304\end{array}$ \\
\hline GEV J2257-2755 & 24.45 & -64.68 & 5.4 & $1.8 \pm 0.6$ & 36 & PKS 2255-282 \\
\hline GEV J1745-3014 & 358.86 & -0.63 & 5.2 & $6.1 \pm 1.3$ & $23^{\mathrm{C}}$ & 3EG J1744-3011 \\
\hline GEV J1228+0159 & 289.89 & 64.30 & 5.2 & $0.9 \pm 0.3$ & $33^{\mathrm{C}}$ & 3 EG J1229+0210, 3C 273 \\
\hline GEV J1824-1511 & 16.36 & -1.03 & 5.1 & $5.6 \pm 1.3$ & $32^{\mathrm{C}}$ & 3EG J1824-1514 \\
\hline GEV J1017-5845 & 284.14 & -1.60 & 4.9 & $4.0 \pm 1.0$ & $33^{\mathrm{C}}$ & 3EG J1013-5915 \\
\hline GEV J2204+4225 & 92.89 & -10.48 & 4.9 & $2.2 \pm 0.7$ & 41 & 3EG J2202+4217, BL Lac \\
\hline GEV J1306-5920 & 304.81 & 3.47 & 4.6 & $2.9 \pm 0.8$ & 29 & $\ldots$ \\
\hline GEV J1230-4839 & 299.39 & 14.07 & 4.5 & $1.6 \pm 0.5$ & 44 & $5.1 \sigma$ in VP 208.0 \\
\hline GEV J0526-6515 & 275.12 & -33.32 & 4.5 & $1.2 \pm 0.4$ & 41 & LMC extended? \\
\hline GEV J2057-4702 & 352.86 & -40.58 & 4.4 & $2.1 \pm 0.8$ & 52 & $\begin{array}{l}\text { 3EG J2055-4716, QSO 2052- } \\
474 ?\end{array}$ \\
\hline GEV J0911+6548 & 148.38 & 38.74 & 4.3 & $1.1 \pm 0.4$ & 59 & 3EG J0910+6556 \\
\hline GEV J2055+2548 & 70.69 & -12.30 & 4.3 & $1.6+0.5$ & 41 & $3 \sigma(0.3-1 \mathrm{GeV})$ \\
\hline GEV J1742-2039 & 6.70 & 4.91 & 4.2 & $2.0 \pm 0.6$ & 24 & 3EG J1741-2050 \\
\hline GEV J1952+3251 & 68.67 & 2.94 & 4.1 & $2.6 \pm 0.8$ & 28 & $3 \sigma(0.3-1 \mathrm{GeV})$, PSR $1951+32$ \\
\hline GEV J0614-3331 & 240.56 & -21.74 & 4.1 & $20+08$ & 57 & 3EG J0616-3310 \\
\hline
\end{tabular}

Most of these new steady sources are in the $3^{\text {rd }}$ EGRET catalog. Of the six sources without a 3EG counterpart, GEV J2257-2755 has been detected above $100 \mathrm{MeV}$ [8] but fell outside the time frame of the $3^{\text {rd }}$ catalog. The source GEV J0526-6515 is very close to the LMC, which is in LM97, and this new source may be more diffuse emission. Above $1 \mathrm{GeV}$, the EGRET point spread may be small enough that the LMC is resolvable. This is being studied further. The source GEV J1952+3251 is consistent with PSR 1951+32, which was previously detected only through pulsar analysis [9] 
but is not in the 3EG catalog. Thus, three of our new sources have no true $100 \mathrm{MeV}$ counterparts. The sources GEV J1306-5920, GEV J1230-4839 and GEV J2055+2548 are all new sources, two of the three being high latitude (biil > 10 degrees).

\section{SEARCH FOR TRANSIENT SOURCES}

Combining the Table 1 sources with those of LM97 gives us a basis for searching for transients. Our approach is to find week time-scale emission by analyzing all 217 public EGRET data sets. For each observation, the sources in LM97 and Table 1 are modeled as part of the background. A map of the residual maximum likelihood is then calculated. This gives the likelihood of a new source being present at each point. There are typically 25000 points per map for a total of 5.6 million likelihood values. The search for transients proceeds by listing points in any map which exceed a $3 \sigma$ significance level. This gives about 3400 points on the sky for all viewing periods. Finally, we correlate all of these features to find instances of $3 \sigma$ excesses within 1 degree of each other (a typical weak point source location radius) in separate but overlapping viewing periods. A new source is indicated by either a high significance in a single viewing period or by repeated outbursts in multiple viewing periods. Finally, we require that the source locations be within the inner 30 degrees of the EGRET field-of-view (16 degrees for EGRET reduced field) to avoid problems with systematic calibration uncertainties in the outer region of the EGRET field [10].

Table 2. Sources of transient GeV gamma rays ordered by galactic longitude.

\begin{tabular}{|c|c|c|c|c|c|c|c|}
\hline Name & LII & BII & $\begin{array}{l}\text { Sig } \\
\text { ma }\end{array}$ & Flux $^{A}$ & $\operatorname{Rad}^{\mathrm{B}}$ & V.P.s & Notes \\
\hline GEV J1653+3945 & 63.58 & $3 \quad 38.97$ & 5.0 & $2.8 \pm 1.0$ & 52 & $201 \& 519$ & Likelihood sum=27.0; MRK 501 \\
\hline GEV J0426+1558 & 179.87 & $7-22.40$ & 3.8 & $4.9 \pm 2.5$ & 57 & $1 \& 616.1$ & $3 \mathrm{EG} \mathrm{J0423+1707}$ \\
\hline GEV J0612+2910 & 182.68 & 5.16 & 4.9 & $12.9 \pm 4.5$ & 56 & $2.1 \& 213$ & Likelihood sum = 29.1 \\
\hline GEV J0448+1054 & 187.76 & $5-21.09$ & 4.3 & $8.0 \pm 3.4$ & 57 & $36.5 \& 39$ & $\begin{array}{l}\text { 3EG J0450+1105; PKS } \\
0446+11\end{array}$ \\
\hline GEV J0339-0144 & 187.93 & $3-42.20$ & 5.6 & $12.0 \pm 4.3$ & 51 & $419.1 \& 420$ & $\begin{array}{l}5.1 \sigma \text { in VP } 420 ; 3 E G, B 0336- \\
019\end{array}$ \\
\hline GEVJ0424-0112 & 195.36 & $6-32.81$ & 5.3 & $5.7 \pm 2.0$ & 27 & 21 & $\begin{array}{l}5.3 \sigma \text { in VP } 21 ; 3 \mathrm{EG}, \text { PKS 0420- } \\
01\end{array}$ \\
\hline GEV J0502-0118 & 200.93 & $3-24.70$ & 5.1 & $8.8 \pm 4.1$ & 56 & 413 & $5.1 \sigma$ in VP $413 ; \mathrm{PKS} 0458-02$ \\
\hline GEV J0638+0446 & 207.19 & $9 \quad-0.74$ & 4.2 & $9.2 \pm 3.1$ & 43 & $1 \& 41$ & 3EG J0634+0521 \\
\hline GEV J1223+2121 & 253.72 & 281.43 & 5.1 & $6.5 \pm 2.7$ & 26 & $311.6 \& 313$ & $\begin{array}{l}\text { 3EG J1224+2118; PKS } \\
1222+21\end{array}$ \\
\hline GEV J1305-8232 & 303.42 & $2-19.68$ & 4.1 & $5.8 \pm 2.5$ & $56^{\mathrm{C}}$ & $17 \& 224$ & 3EG J1249-8330 \\
\hline GEV J1409-6126 & 312.13 & 0.02 & 5.1 & $15.6 \pm 4.0$ & 26 & $314 \& 424$ & $3 E G \mathrm{~J} 1410-6147$ \\
\hline GEV J1715-4044 & 346.77 & $\begin{array}{ll}7 & -1.36\end{array}$ & 4.3 & $19.3 \pm 6.3$ & 37 & $334 \& 423$ & Likelihood sum $=27.9$ \\
\hline GEV J1323+2206 & 359.91 & 181.20 & 5.8 & $10.1 \pm 3.8$ & 22 & $308 \& 313$ & $\begin{array}{l}5.3 \sigma \text { in VP } 8.0 ; 3 \mathrm{EG} \\
\mathrm{B} 1324+2226\end{array}$ \\
\hline
\end{tabular}


To date, two sets of simulations corresponding to the same exposures and sky pointings as the 217 actual data sets have been calculated. These simulations indicate that for a source detected in a single viewing period, a maximum likelihood statistic of $22(4.7 \sigma)$ is adequate to ensure less than one chance detection. For repeated outbursts, the cleanest separation of signal from chance coincidences comes from summing the likelihood values for the coincident pairs. For now, we concentrate on sources detected in two viewing periods, for which simulations indicate that a sum of the two likelihoods above 26 yields at most a single spurious detection. We also include sources that are detected at $3 \sigma$ in at least two viewing periods and correspond to a 100 $\mathrm{MeV}$ catalog source regardless of the summed value.

The thirteen sources listed in Table 2 satisfy at least one of the three selection criteria. Four of the sources exceed $4.7 \sigma$ in a single viewing period while three are detected only by summing likelihoods. Eight of these 13 transient sources have counterparts in the $3^{\text {rd }}$ EGRET catalog, for which 6 are included solely on the basis of this association. The values listed in Table 2 are for the most significant detection, which may be the sum of two viewing periods even for those sources that qualify by
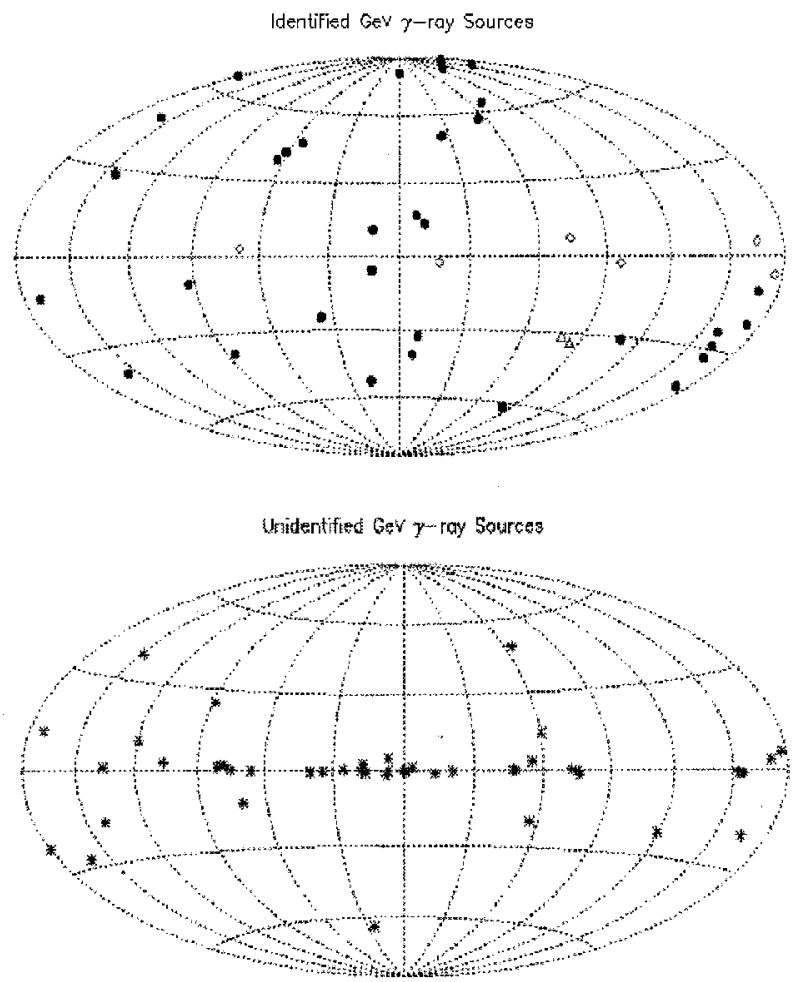

Figure 1. The positions of identified and unidentified GeV gamma-ray sources are plotted in galactic coordinates. These plots contain sources from LM97 and from this paper and the distributions are strikingly different. 
being significantly detected in a single viewing period. The viewing periods used to calculate the source parameters are listed in the "VP" column. Two of the most interesting detections are of GEV J0502-0118 which is associated with a 1 Jy radio source and the TeV detected MRK 501 which has only recently been discovered to emit in the EGRET data [11].

\section{DISCUSSION}

A preliminary analysis of data above $1 \mathrm{GeV}$ finds 29 new sources of $\mathrm{GeV}$ gammaray emission, 13 of which are found in a search for transient emission. Six of these sources are previously unreported. Of the 13 sources detected in the transient search, four are low latitude. These could be high-energy analogs to the $100 \mathrm{MeV}$ transient GRO J1838-04 [12]. Many of the new sources have bright radio sources in their error circles, although most of the radio sources have $4.8 \mathrm{GHz}$ fluxes below $1 \mathrm{Jy}$. Future work will emphasize other candidates, class studies, and comparing $\mathrm{GeV}$ and 100 $\mathrm{MeV}$ sources. Special attention will be paid to the unidentified sources, which as Figure 1 shows, tend to be a galactic population.

\section{REFERENCES}

1. Bradbury, S.M., et al. 1997, A\&A, 320, L5

2. Catanese, M., et al. 1998, ApJ, 501, 616

3. Tanimori, T., et al. 1998, ApJ, 497, L25

4. Lamb, R.C. \& Macomb, D.J. 1997, ApJ 490, 493 (LM97)

5. Hartman, R.C., et al. 1999, ApJS, 123, 79

6. Mattox, J.R. et al. 1996, ApJ, 461, 396

7. Bertsch, D.L., et al. 1993, ApJ, 416, 587

8. Macomb, D.J., Gehrels, N. \& Shrader, C.R. 1999, ApJ, 513, 652

9. Ramanamurthy, P.V., et al. 1995, ApJ, 450, 791

10. Esposito, J.A., et al. 1999, ApJS, 123, 207

11. Sreekumar, P. et al. 1999, American Astronomical Society, HEAD meeting \#31, \#03.03

12. Tavani, M., et al. 1997, ApJ, 479, L109 